\title{
Genomic Earthquakes in the Human Germline and Their Ramifications
}

The minimal requirement for a complex chromosome rearrangement (CCR) is that it involves 3 or more chromosome breaks [Madan, 2013; Poot and Haaf, 2015]. This definition was based on decades of examining karyotypes through a microscope. With the advent of molecular cytogenetic techniques, the resolution of the "molecular" karyotype improved, and consequently, the number of detected chromosomal breaks increased. Reexamination of "old" cases revealed that the chromosomal breaks were often acompanied by rearrangements at the breakpoints and also at remote sites in the genome [Kloosterman et al., 2011, 2012; Chiang et al., 2012; Gu et al., 2013; Nazaryan et al., 2014]. Thus, some cases of chromoplexy, translocations involving 3 or more chromosomes, became much more complex [Zhang et al., 2013; Nazaryan and Tommerup, 2016].

During meiosis, chromosomes form pairs. In cases of chromoplexy, this pairing involved formation of complexes with 3, 4, or even more participating chromosomes. Subsequently, these hexavalent, octavalent, and even higher valence chiasmata need to be resolved, such that the translocation chromosomes can separate during the next metaphase. Although the mechanisms of this process are not fully understood, one can assume that the DNA helices need to unwind locally and that doublestrand breaks have to form. Conceivably, macromolecular steric hinderance will occur, and the involved DNA molecules will move relative to each other analogous to the rubbing of tectonic plates during an earthquake. Thus, it is likely that at the breakpoints, a small number of nucleotides will be lost. During a subsequent round of DNA replication, this loss will become a small deletion. Alternatively, the lost stretches of DNA may be replicated autonomously and become reintegrated in the chromosome at the original breakpoint or at a remote site. This has indeed been demonstrated for a case of germline chromothripsis [Slamova et al., 2018]. In this case, joining of the DNA fragments proceeded by canonical NHEJ and alt-NHEJ concomitantly. This suggests that the WRN-encoded $3^{\prime} \rightarrow 5^{\prime}$ exonuclease $3^{\prime} \rightarrow 5^{\prime}$ helicase, which governs preference for either form of NHEJ, may operate on a siteby-site basis.

Taken together, the "molecular signatures" at the breakpoints in cases of chromoplexy closely resemble those remaining after chromothripsis [Poot, 2018]. Distinguishing chromoplexy from chromothripsis may become even more difficult if one considers a hypothetical mechanism proposed by Zhang et al., [2013]. These authors noted that the pairs of adjacent breakpoint ends are less than $1 \mathrm{kbp}$ apart, such that the translocations were almost certainly chained together. If the first chain of 3 translocations occurred all at once, this would have resulted in a functional centromere-containing derivative chromosome. This is retained in the final chromosomal configuration together with 2 mitotically unstable derivatives, which would either be lost or undergo additional rearrangements. The authors suggest that unstable chromosomes generated by a chain of translocations (chromoplexy) could result in subsequent catastrophes adding chromothripsis on top of the original translocations [Zhang et al., 2013]. They termed this model translocation-induced chromothripsis. However plausible such a process based on macromolecular steric hinderance may seem, other, equally plausible, mechansisms of chromothripsis such as micronucleus-based and TREX1 exonuclease-mediated fragmentation of chromosome bridges have been demonstrated [Ly and Cleveland, 2017; Poot, 2018].

\section{KARGER}

(c) 2019 S. Karger AG, Basel

E-Mail karger@karger.com

www.karger.com/msy
Martin Poot

Department of Human Genetics, Universtity of Würzburg

Biozentrum, Am Hubland

DE-97074 Würzburg (Germany)

E-Mail Martin_Poot@hotmail.com 
A recent study of 83 breakpoint-junctions from 21 cases in which more than one CNV arose simultaneously on a single chromosome indicated that the cell may resort to 3 different types of response [Nazaryan-Petersen et al., 2018]: First, cases with only deletions, but often additional structural rearrangements, such as insertions and inversions, which could be a result of multiple double-strand breaks followed by nonhomologous repair. Second, cases with only duplications or combinations of deletions and duplications, which contained mostly interspersed duplications and breakpoint-junctions enriched with microhomology, which is consistent with serial template switching during DNA replication (chromoanasynthesis). Third, rearrangements that were repeat mediated [NazaryanPetersen et al., 2016]. Thus, multiple CNVs clustered on a single chromosome may arise through either chromothripsis or chromoanasynthesis. The latter resembles the mechanism described for de novo duplications or deletions at the 17p11.2 locus in patients with Potocki-Lupski syndrome or Smith-Magenis syndrome [Beck et al., 2019]. These appear to have resulted from error prone microhomology-mediated break-induced repair (MMBIR) of double-strand breaks. Interestingly, within $1 \mathrm{Mbp}$ from the breakpoints, a significantly elevated level of single nucleotide variants was found. This is reminiscent of kataegis, which accompanies chromothripsis after TREX1 exonuclease-mediated fragmentation of chromosome bridges [Maciejowski et al., 2015].

While cases of chromoplexy or clustered CNVs are rare findings, 2 recent reports describe even more complicated and rarer genome rearrangements [Liu et al., 2017; Hattori et al., 2019]. In a cohort of 60,000 individuals analyzed by genome-wide array CGH, Liu et al. [2017] identified 1,437 individuals with one de novo CNV, 39 with two, 1 with three, 1 with four, and 1 with seven de novo CNVs. These cases are characterized by CCRs, microhomology, and templated insertions at the breakpoints as well as de novo single nucleotide variants in close proximity to breakpoint junctions, which is consistent with a fork stalling and template switching/MMBIR mechanism. The CNVs are predominantly copy number gains (duplications and triplications), which often occurred in tandem. Since all cells in the body were affected, these multiple de novo CNVs (mdnCNVs) must have arisen in a perizygotic interval. Contrary to the "mutator phenotype" in cancer, the driving force behind the mdnCNVs must have been lost after the perizygotic stage, since no further rearrangements leading to somatic mosaicism were detected [Loeb, 2001; Liu et al., 2017]. Thus far, one can only speculate as to the molecular nature of the driv- ing force behind these mdnCNVs, which most likely arose before the end of meiosis II.

A third case of a higly intricate CCR is clearly distinct from the previously discussed mdnCNVs [Hattori et al., 2019]. Of the 5 affected chromosomes, 2 underwent chromothripsis, a third one an inversion, the fourth and the fifth a tandem duplication, and a deletion with an inverted duplication; each was followed by homologous recombination. The duplicated regions contained both diallelic and triallelic segments, indicating that these genomic rearrangements arose during premeiotic mitosis and were subsequently modified by crossovers during meiosis I. The breakpoints of the rearrangements showed signatures of NHEJ, replication-based errors, and chromothripsis. The rearrangements took place in the paternal germline and were independent of local DNA motifs, which is clearly distinct from the described cases of mdnCNVs in which most rearrangements took place in primary oocytes and postzygotic cells [Liu et al., 2017]. None of the breakpoints in this case were accompanied by de novo single nucleotide variants, as in the previously reported cases of mdnCNVs [Liu et al., 2017]. These characteristics indicate that during spermatogenesis, a transient multifocal genomic crisis can introduce concomitantly multiple chromothriptic and non-chromothriptic rearrangements into the genome. This case broadens the spectrum of mdnCNVs and sets it apart from the "genome chaos" frequently observed in malignant disease [Liu et al., 2014].

Regardless of the multiple, sometimes concomitantly operating, mechanisms provoking such genomic earthquakes, medical genetics is also concerned with the functional consequences of such extremely complicated CCRs. Some $70 \%$ of the microscopically detectable CCRs are without phenotypic effect [Pellestor et al., 2011]. Even a germline chromothriptic event, which disrupted 6 protein encoding genes, stably segregated in 11 healthy siblings through 3 generations [Bertelsen et al., 2016]. This family illustrated that heterozygous gene disruptions, involving gene truncation or gene fusion, are not necessarily pathogenic. To decipher the putative phenotypic impact of gene disruptions, the presence of chromatin modifications, as marks for active or silenced genes in the vicinity of the affected genes, have been proposed [Poot and Haaf, 2015]. While this approach may indicate which gene disruptions may be worthwhile of further study, it remains a bioinformatic prediction in need of experimental corroboration.

To resolve a case of Moebius syndrome with a CCR, summarized as $46, \mathrm{XY}, \mathrm{t}(7 ; 8 ; 11 ; 13)$, that involved 41 clustered breakpoints, indicating chromothripsis, and trun- 
cating 12 protein-encoding genes, a novel approach has been taken [Nazaryan-Petersen et al., 2019]. Starting from the 2 known genes for Moebius syndrome in which mutations have been confirmed (PLXND1 and REV3L), the authors interrogated their 12 disrupted genes in silico for possible interactions with these genes based on experiments, coexpression studies, curated databases, and text mining. Among the 12 truncated protein-coding genes, SEMA3A (sema domain, immunoglobulin domain [Ig], short basic domain secreted, semaphorin $3 \mathrm{~A}$ ) is known to bind to PLXND1 and PIK3CG (phosphatidylinositol-4,5bisphosphate 3-kinase, catalytic subunit $\gamma$ ) to REV3L. This in silico interaction study revealed a functional networkencompassing 4 truncated genes, including SEMA3A, SEMA3D (sema domain, immunoglobulin domain [Ig], short basic domain, secreted, semaphorin 3D), PIK3CG, and UBR5 (ubiquitin protein ligase E3 component $\mathrm{n}$ - recognin 5). Literature on the functions of these genes further supported their possible involvement in Moebius syndrome. Based on the 4 genes making up this functional network, the in silico interaction study further suggested 6 more candidate genes for Moebius syndrome [Nazaryan-Petersen et al., 2019]. A similar approach has recently been taken with regard to genes affected by CNVs in patients with nonsyndromic forms of craniosynostosis [Poot, 2019]. Some of those were found to form a functional network with genes associated with monogenic craniosynostosis syndromes. Searching for functional interactions of genes, being disrupted or lost in sporadic, nonsyndromic patients, with genes in syndromic forms of disease may prove helpful for identification of novel candidate genes and for broadening our understanding of the underlying biology of genetic disease in the future.

Martin Poot

\section{References}

Beck CR, Carvalho CMB, Akdemir ZC, Sedlazeck FJ, Song X, et al: Megabase length hypermutation accompanies human structural variation at 17p11.2. Cell 176:1310-1324 (2019).

Bertelsen B, Nazaryan-Petersen L, Sun W, Mehrjouy MM, Xie G, et al: A germline chromothripsis event stably segregating in 11 individuals through three generations. Genet Med 18:494-500 (2016).

-Chiang C, Jacobsen JC, Ernst C, Hanscom C, Heilbut A, et al: Complex reorganization and predominant non-homologous repair following chromosomal breakage in karyotypically balanced germline rearrangements and transgenic integration. Nat Genet 44:390-397 (2012).

Gu H, Jiang JH, Li JY, Zhang YN, Dong XS, et al: A familial Cri-du-Chat/5p deletion syndrome resulted from rare maternal complex chromosomal rearrangements (CCRs) and/or possible chromosome $5 \mathrm{p}$ chromothripsis. PLoS One 8:e76985 (2013)

Hattori A, Okamura K, Terada Y, Tanaka R, Katoh-Fukui Y, et al: Transient multifocal genomic crisis creating chromothriptic and non-chromothriptic rearrangements in prezygotic testicular germ cells. BMC Med Genomics 12:77 (2019).

Kloosterman WP, Guryev V, van Roosmalen M, Duran KJ, de Bruijn E, et al: Chromothripsis as a mechanism driving complex de novo structural rearrangements in the germline. Hum Mol Genet 20:1916-1924 (2011).

-Kloosterman WP, Tavakoli-Yaraki M, van Roosmalen MJ, van Binsbergen E, Renkens I, et al: Constitutional chromothripsis rearrangements involve clustered double-stranded DNA breaks and nonhomologous repair mechanisms. Cell Rep 1:648-655 (2012).
Liu G, Stevens JB, Horne SD, Abdallah BY, Ye KJ, et al: Genome chaos: survival strategy during crisis. Cell Cycle 13:528-537 (2014).

Liu P, Yuan B, Carvalho CMB, Wuster A, Walter $\mathrm{K}$, et al: An organismal CNV mutator phenotype restricted to early human development. Cell 168:830-842.e7 (2017).

Loeb LA: A mutator phenotype in cancer. Cancer Res 61:3230-3239 (2001).

Ly P, Cleveland DW: Rebuilding chromosomes after catastrophe: emerging mechanisms of chromothripsis. Trends Cell Biol 27:917-930 (2017).

-Maciejowski J, Li Y, Bosco N, Campbell PJ, de Lange T: Chromothripsis and kataegis induced by telomere crisis. Cell 163:1641-1654 (2015).

Madan K: What is a complex chromosome rearrangement? Am J Med Genet A 161A:11811184 (2013).

- Nazaryan L, Stefanou EG, Hansen C, Kosyakova $\mathrm{N}, \mathrm{Bak} \mathrm{M}$, et al: The strength of combined cytogenetic and mate-pair sequencing techniques illustrated by a germline chromothripsis rearrangement involving FOXP2. Eur J Hum Genet 22:338-343 (2014).

-Nazaryan-Petersen L, Tommerup N: Chromothripsis and human genetic disease. eLS 1-10 (2016).

- Nazaryan-Petersen L, Bertelsen B, Bak M, Jonson L, Tommerup N, et al: Germline chromothripsis driven by L1-mediated retrotransposition and Alu/Alu homologous recombination. Hum Mutat 37:385-395 (2016).
Nazaryan-Petersen L, Eisfeldt J, Pettersson M, Lundin J, Nilsson D, et al: Replicative and non-replicative mechanisms in the formation of clustered CNVs are indicated by whole genome characterization. PLoS Genet 14: e1007780 (2018).

- Nazaryan-Petersen L, Oliveira IR, Mehrjouy MM, Mendez JMM, Bak M, et al: Multigenic truncation of the semaphoring-plexin pathway by a germline chromothriptic rearrangement associated with Moebius syndrome. Hum Mut 40:1057-1062 (2019).

-Pellestor F, Anahory T, Lefort G, Puechberty J, Liehr T, et al: Complex chromosomal rearrangements: origin and meiotic behavior. Hum Reprod Update 17:476-494 (2011).

- Poot M: Genes, proteins, and biological pathways preventing chromothripsis. Methods $\mathrm{Mol}$ Biol 1769:231-251 (2018).

Poot M: Structural genome variations related to craniosynostosis. Mol Syndromol 10:24-39 (2019).

Poot M, Haaf T: Mechanisms of origin, phenotypic effects and diagnostic implications of complex chromosome rearrangements. Mol Syndromol 6:110-134 (2015).

-Slamova Z, Nazaryan-Petersen L, Mehrjouy MM, Drabova J, Hancarova $M$, et al: Very short DNA segments can be detected and handled by the repair machinery during germline chromothriptic chromosome reassembly. Hum Mutat 39:709-716 (2018).

Zhang CZ, Leibowitz ML, Pellman D: Chromothripsis and beyond: rapid genome evolution from complex chromosomal rearrangements. Genes Dev 27:2513-2530 (2013). 